\title{
Boundary element modeling of complex grounding systems: study on current distribution
}

\author{
D. Poljak ${ }^{1}$, D. Ćerdic ${ }^{2}$, V. Doric ${ }^{1}$, A. Peratta ${ }^{2}$, \\ V. Roje ${ }^{1} \&$ C. A. Brebbia ${ }^{2}$ \\ ${ }^{1}$ University of Split, Croatia \\ ${ }^{2}$ Wessex Institute of Technology, UK
}

\begin{abstract}
The assessment of current distribution induced along complex grounding systems has been undertaken using the corresponding antenna model and the set of Pocklington integro-differential equations for curved wires. The set of Pocklington equations is numerically handled via the Galerkin-Bubnov scheme of the Indirect Boundary Element Method (GB-IBEM) featuring the isoparametric elements. Some illustrative numerical results for the current distribution are presented in the paper.
\end{abstract}

Keywords: boundary elements, antenna theory, set of Pocklington equations, grounding systems.

\section{Introduction}

The analysis of complex grounding systems being important component in lightning protection systems (LPS) is of great interest in electromagnetic compatibility (EMC) and high voltage (HV) engineering. In general, grounding systems can be analyzed using the transmission line model (TLM) [1,2], which principle feature is simplicity, or the antenna model (AM) [3-5], which is considered to be the rigorous approach, (but at the same time much more demanding, regarding both the formulation and numerical solution. The keyparameter in the antenna model is the equivalent current distribution flowing along grounding structures. Given the knowledge of the current distribution along the grounding system it is possible to determine other parameters of 
interest, i.e. the scattered voltage, the input impedance and the transient impedance.

This paper represents an extension of the previous work of the authors on the subject which was related to the BEM analysis of a single grounding electrode $[6,7]$. The antenna model of the grounding systems composed from straight conductors is based on the set of Pocklington's integro-differential equations for curved wires. The effect of a lossy ground is taken into account via the corresponding reflection coefficient [5] thus avoiding the rigorous approach based on the analytically demanding and numerically time consuming Sommerfeld integrals [8]. The set of Pocklington equations is numerically treated by means of the Galerkin-Bubnov scheme of the Boundary Element Method (GB-IBEM) with isoparametric elements [9]. Some illustrative numerical results for the current distribution along different grounding geometries are presented.

\section{Wire grid model of the grounding system}

The geometry of interest, shown in Fig 1, is the complex grounding system composed from interconnected conductors.

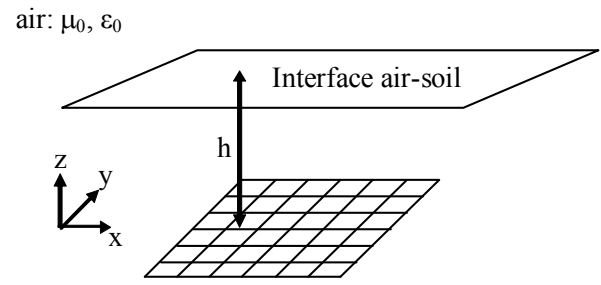

soil: $\mu, \rho, \varepsilon$

Figure 1: Geometry of a complex grounding system.

The set of Pocklington equiations for a configuration of interconnected buried wires can be obtained as an extension of the Pocklington equation for a single buried wire of arbitrary shape [7].

The Pocklington equation for a single grounding electrode can be derived by enforcing the continuity conditions for the tangential components of the electric field along the perfectly conducting (PEC) wire surface. An extension to the case of imperfectly conducting wire is straightforward $[6,7]$. For the PEC wire the total field composed from the excitation field $\vec{E}^{\text {exc }}$ and scattered field $\vec{E}^{s c t}$ vanishes:

$$
\vec{e}_{x} \cdot\left(\vec{E}^{e x c}+\vec{E}^{s c t}\right)=0 \text { on the wire surface }
$$

Starting from Maxwell's equations and Lorentz gauge the scattered electric field can be expressed in terms of the vector potential $\vec{A}$ : 


$$
\vec{E}^{s c t}=-j \omega \vec{A}+\frac{1}{j \omega \mu \varepsilon} \nabla(\nabla \vec{A})
$$

which is defined by the particular integral:

$$
\vec{A}(s)=\frac{\mu}{4 \pi} \int_{C} I\left(s^{\prime}\right) g\left(s, s^{\prime}, s^{*}\right) \vec{s}^{\prime} d s^{\prime}
$$

where $I\left(\mathrm{~s}^{\prime}\right)$ is the induced current along the wire, and $g\left(\mathrm{~s}, \mathrm{~s}^{\prime}\right)$ is the corresponding Green's function of the form:

$$
g\left(s, s^{\prime}, s^{*}\right)=g_{0}\left(s, s^{\prime}\right)-\Gamma_{r e f} g_{i}\left(s, s^{*}\right)
$$

where $g_{0}\left(s, s^{\prime}\right)$ denotes the lossy medium Green function, while $g_{i}\left(s, s^{\prime}\right)$ arises from the image theory. These functions are given by:

$$
g_{0}\left(s, s^{\prime}\right)=\frac{e^{-\gamma R_{1}}}{R_{1}} \quad g_{i}\left(s, s^{\prime}\right)=\frac{e^{-\gamma R_{2}}}{R_{2}}
$$

and $R_{1}$ and $R_{2}$ are the distances from the source point to the observation point, respectively, while the propagation constant of the lower medium is defined as:

$$
\gamma=\sqrt{j \omega \mu \sigma-\omega^{2} \mu \varepsilon_{r g} \varepsilon_{0}}
$$

where $\varepsilon_{r g}$ and $\sigma$ are the corresponding ground permittivity and conductivity, respectively.

The influence of a ground-air interface is taken into account via the reflection coefficient of the form [6]:

$$
\Gamma_{r e f}=\frac{\frac{1}{n} \cos \theta-\sqrt{\frac{1}{n}-\sin ^{2} \theta}}{\frac{1}{n} \cos \theta+\sqrt{\frac{1}{n}-\sin ^{2} \theta}} ; \theta=\operatorname{arctg} \frac{\left|x-x^{\prime}\right|}{2 d} ; \underline{n}=\frac{\varepsilon_{\text {eff }}}{\varepsilon_{0}}
$$

The complex permittivity of the lossy ground $\varepsilon_{\text {eff }}$ is given by:

$$
\varepsilon_{e f f}=\varepsilon_{r} \varepsilon_{0}-j \frac{\sigma}{\omega}
$$

Combining equations (1)-(3) and performing some mathematical manipulation leads to the integro-differential equation of the Pocklington type for the unknown current distribution induced along the thin wire of an arbitrary shape buried in a lossy half-space [9]:

$$
\begin{aligned}
& E^{e x c}(s)=-\frac{1}{j 4 \pi \omega \varepsilon_{e f f}} \int_{C^{\prime}} I\left(s^{\prime}\right) \cdot \vec{s} \cdot \vec{s}^{\prime} \cdot\left[\frac{\partial^{2}}{\partial s \partial s^{\prime}}-\gamma^{2}\right] g_{0}\left(s, s^{\prime}\right) d s^{\prime}+ \\
& +\Gamma_{r e f} \int_{C^{\prime}} I\left(s^{\prime}\right) \cdot \vec{s}^{\prime} \cdot \vec{s}^{*} \cdot\left[\frac{\partial^{2}}{\partial s \partial s^{*}}-\gamma^{2}\right] g_{i}\left(s, s^{*}\right) d s^{\prime}
\end{aligned}
$$


An extension to the case of multiple wires buried in a lossy medium is straightforward and results to the set of coupled Pocklington equations:

$$
E_{m}^{e x c}(s)=-\frac{1}{j 4 \pi \omega \varepsilon_{e f f}} \sum_{n=1}^{M}\left[\begin{array}{l}
\int_{C^{\prime}} I_{n}\left(s_{n}^{\prime}\right) \cdot \vec{s}_{n} \cdot \vec{s}_{n}^{\prime} \cdot\left[\frac{\partial^{2}}{\partial s_{m} \partial s_{n}^{\prime}}-\gamma^{2}\right] g_{0, n m}\left(s_{m}, s_{n}^{\prime}\right) d s^{\prime}+ \\
\left.+\Gamma_{r e f} \int_{C^{\prime}} I_{n}\left(s^{\prime}\right) \cdot \vec{s}_{m} \cdot \vec{s}_{n}^{*} \cdot\left[\frac{\partial^{2}}{\partial s_{m} \partial s_{n}^{*}}-\gamma^{2}\right] g_{i, n m}\left(s_{m}, s_{n}^{*}\right) d s^{\prime}\right]
\end{array}\right], m=1,2, \ldots M
$$

where $I_{n}\left(s^{\prime}\right)$ is unknown current distribution along the $n$-th wire, $E_{m}^{e x c}(s)$ the excitation function on the $m$-th wire.

Finally, the grounding system is energized by the injection of the current source with one terminal connected to the grounding structure and the other one grounded at infinity [4]. Consequently, the left hand side of the equation (10) vanishes reducing the corresponding Pocklington equation to the homogenous one.

$$
-\frac{1}{j 4 \pi \omega \varepsilon_{\text {eff }}} \sum_{n=1}^{M}\left[\begin{array}{l}
\int_{C^{\prime}} I_{n}\left(s_{n}^{\prime}\right) \cdot \vec{s}_{n} \cdot \vec{s}_{n}^{\prime} \cdot\left[\frac{\partial^{2}}{\partial s_{m} \partial s_{n}^{\prime}}-\gamma^{2}\right] g_{0, n m}\left(s_{n}, s_{n}^{\prime}\right) d s^{\prime}+ \\
+\Gamma_{r e f} \int_{C^{\prime}} I_{n}\left(s^{\prime}\right) \cdot \vec{s}_{m} \cdot \vec{s}_{n}^{*} \cdot\left[\frac{\partial^{2}}{\partial s_{m} \partial s_{n}^{*}}-\gamma^{2}\right] g_{i, n m}\left(s_{m}, s_{n}^{*}\right) d s^{\prime}
\end{array}\right]=0, \quad m=1,2, \ldots M
$$

This current source excitation is incorporated into formulation through the boundary conditions.

\section{Boundary element procedures}

The set of Pocklington integro-differential equations (11) is numerically handled by means of the Galerkin-Bubnov variant of Indirect Boundary Element Method (GB-IBEM). The Boundary Element solution technique used in this work is an extension of the method applied to single wire cases reported elsewhere, e.g. in $[6,7,9]$.

The unknown current along the $n$-th wire segment is expressed by the sum of a linearly independent basis functions $f_{n i}$, with unknown complex coefficients $I_{n i}$ :

$$
I_{n}^{e}\left(s^{\prime}\right)=\sum_{i=1}^{n} I_{n i} f_{n i}\left(s^{\prime}\right)=\{f\}_{n}^{T}\{I\}_{n}
$$

and the use of isoparametric elements yields:

$$
I_{n}^{e}(\zeta)=\sum_{i=1}^{n} I_{n i} f_{n i}(\zeta)=\{f\}_{n}^{T}\{I\}_{n}
$$

where $N_{\mathrm{e}}$ is the number of local nodes on the element.

A linear approximation over a segment along $n$-the wire is used in this work as this choice was proved to be optimal in modeling of various wire structures [9]. The corresponding shape functions are given by:

$$
f_{i}=\frac{1-\zeta}{2} \quad f_{i+1}=\frac{1+\zeta}{2}
$$

Applying the weighted residual approach with Galekin-Bubnov procedure (same test and basis functions) after performing a certain mathematical 
manipulations, the following matrix equation is obtained the set of Pocklington equations is transformed into a system of algebraic equations:

$$
\sum_{n=1}^{N_{w}} \sum_{i=1}^{N_{g}}[Z]_{m n}^{j i}\{I\}_{\mathrm{ni}}=0, \quad \mathrm{~m}=1,2, \ldots, N_{w}
$$

where $N_{\mathrm{w}}$ is the total number of wires, $N_{g}$ is total number of elements along the wire grid. Mutual impedance $[Z]_{m n}^{j i}$ represents the interaction between different wire segments is given by:

$$
[Z]_{m n}^{j i}=-\frac{1}{j 4 \pi \omega \varepsilon_{e f f}}\left[\begin{array}{l}
\int_{-1}^{1} \int_{-1}^{1}\{D\}_{j}\left\{D^{\prime}\right\}_{i}^{T} g_{0, m n}\left(s_{m}, s_{n}^{\prime}\right) d s_{n}^{\prime} d s_{m}+ \\
+k_{1}^{2} \int_{-1}^{1} \int_{-1}^{1} \vec{s}_{m} \cdot \vec{s}_{n}^{\prime} \cdot\{f\}_{j}\left\{f^{\prime}\right\}_{i}^{T} g_{0, m n}\left(s_{m}, s_{n}^{\prime}\right) d s_{n}^{\prime} d s_{m} \\
-\Gamma_{r e f} \int_{-1}^{1} \int_{-1}^{1}\{D\}_{j}\left\{D^{\prime}\right\}_{i}^{T} g_{i, m n}\left(s_{m}, s_{n}^{*}\right) d s_{n}^{\prime} d s_{m}^{*}+ \\
+\Gamma_{r e f} k_{1}^{2} \int_{-1}^{1} \int_{-1}^{1} \vec{s}_{m} \cdot \vec{s}_{n}^{*} \cdot\{f\}_{j}\left\{f^{\prime}\right\}_{i}^{T} g_{i, m n}\left(s_{m}, s_{n}^{*}\right) d s_{n}^{\prime} d s_{m}^{*}
\end{array}\right]
$$

Matrices $\{f\}$ and $\left\{f^{\prime}\right\}$ contain the shape functions while $\{D\}$ and $\left\{D^{\prime}\right\}$ contain their derivatives, and $\Delta l_{i}, \Delta l_{j}$ are the widths of $i$-th and $j$-th boundary elements. The implementation of isoparametric elements gives:

$$
[Z]_{m n}^{j i}=-\frac{1}{j 4 \pi \omega \varepsilon_{e f f}}\left[\begin{array}{l}
\int_{-1}^{1} \int_{-1}^{1}\{D\}_{j}\left\{D^{\prime}\right\}_{i}^{T} g_{0, m n}\left(s_{m}, s_{n}^{\prime}\right) \frac{d s_{n}^{\prime}}{d \zeta^{\prime}} d \zeta^{\prime} \frac{d s_{m}}{d \zeta} d \zeta+ \\
+k_{1}^{2} \int_{-1}^{1} \int_{-1}^{1} \vec{s}_{m} \cdot \vec{s}_{n}^{\prime} \cdot\{f\}_{j}\left\{f^{\prime}\right\}_{i}^{T} g_{0, m n}\left(s_{m}, s_{n}^{\prime}\right) \frac{d s_{n}^{\prime}}{d \zeta^{\prime}} d \zeta^{\prime} \frac{d s_{m}}{d \zeta} d \zeta \\
-\Gamma_{r e f} \int_{-1}^{1} \int_{-1}^{1}\{D\}_{j}\left\{D^{\prime}\right\}_{i}^{T} g_{i, m n}\left(s_{m}, s_{n}^{*}\right) \frac{d s_{n}^{\prime}}{d \zeta^{\prime}} d \zeta^{\prime} \frac{d s_{m}}{d \zeta} d \zeta+ \\
+\Gamma_{r e f} k_{1}^{2} \int_{-1}^{1} \int_{-1}^{1} \vec{s}_{m} \cdot \vec{s}_{n}^{*} \cdot\{f\}_{j}\left\{f^{\prime}\right\}_{i}^{T} g_{i, m n}\left(s_{m}, s_{n}^{*}\right) \frac{d s_{n}^{\prime}}{d \zeta^{\prime}} d \zeta^{\prime} \frac{d s_{m}}{d \zeta} d \zeta
\end{array}\right]
$$

The current source $I_{\mathrm{g}}$ is taken into account as a forced boundary condition at the certain node $i$ of the grounding system:

$$
I_{i}=I_{g}
$$

The treatment of wire junctions is related to the Kirchhoff's current law in its integral and differential form, respectively, related to continuity of currents and charges at the junction.

\section{Numerical results}

Some typical grounding configurations regarding two-wire junctions and threewire junctions are analyzed. In all cases the unit current source is given by: 
128 Boundary Elements and Other Mesh Reduction Methods XXXII

$$
I_{g}=1 e^{j 0}
$$

\subsection{Two-wire junction}

The grounding system in the form of two wire junction is shown in Fig. 2. In Figs 3 to 7 the current distribution along the two horizontal grounding electrodes is presented.

Fig. 3 is related to two $6 \mathrm{~m}$ long electrodes $\left(L_{1}=L_{2}=6 \mathrm{~m}\right)$, with radius $a=5 \mathrm{~mm}$, the burial depth $d=0.5 \mathrm{~m}$, ground permittivity $\varepsilon_{\mathrm{r}}=10$, and specific resistance $\rho=$ of $100 \Omega \mathrm{m}$. The frequency is $f=10 \mathrm{MHz}$.

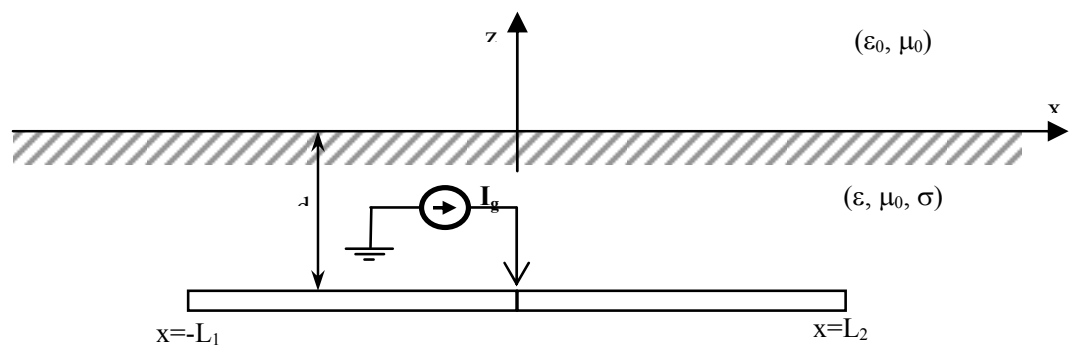

Figure 2: Two-wire junction energized by the current source.

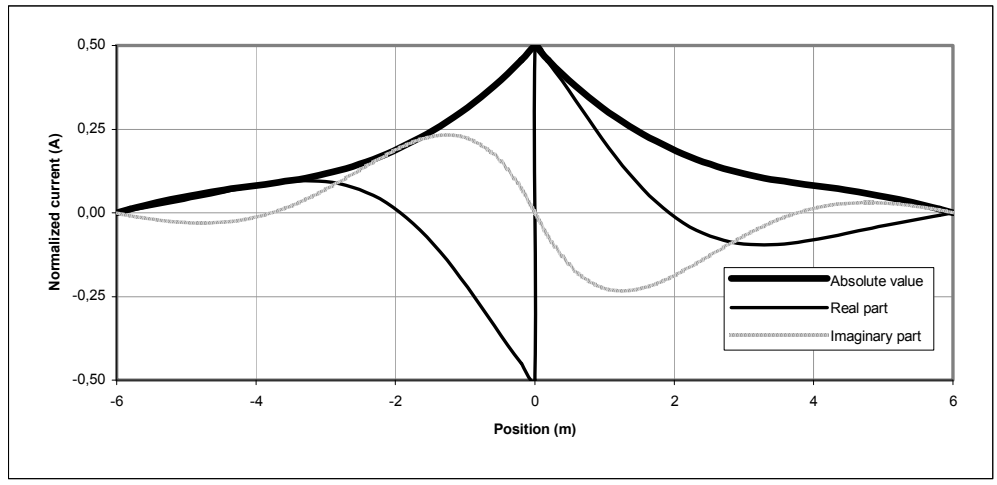

Figure 3: The current distribution along the two-wire junction: $L_{1}=6 \mathrm{~m}$, $L_{2}=6 \mathrm{~m}, d=0.5 \mathrm{~m}, \varepsilon_{\mathrm{r}}=10, \rho=100 \Omega \mathrm{m}, f=10 \mathrm{MHz}$.

Fig. 4 shows the current distribution along the two electrodes of different length $\left(L_{1}=4 \mathrm{~m}, L_{2}=8 \mathrm{~m}\right)$ while the other parameters are the same. 
Figure 5 shows the induced current distribution along the two $6 \mathrm{~m}$ long electrodes for the case of higher specific resistance $\rho=5400 \Omega \mathrm{m}$, while the other parameters are the same.

Figures 6 and 7 show the induced current distribution along the two electrodes of different length; $L_{1}=4 \mathrm{~m}, L_{2}=8 \mathrm{~m}$, and $L_{1}=3 \mathrm{~m}, L_{2}=9 \mathrm{~m}$, the specific resistance is $\rho=5400 \Omega \mathrm{m}$, while the other parameters are the same.

The effect of the different electrode lengths and different values of ground specific resistance is clearly visible.

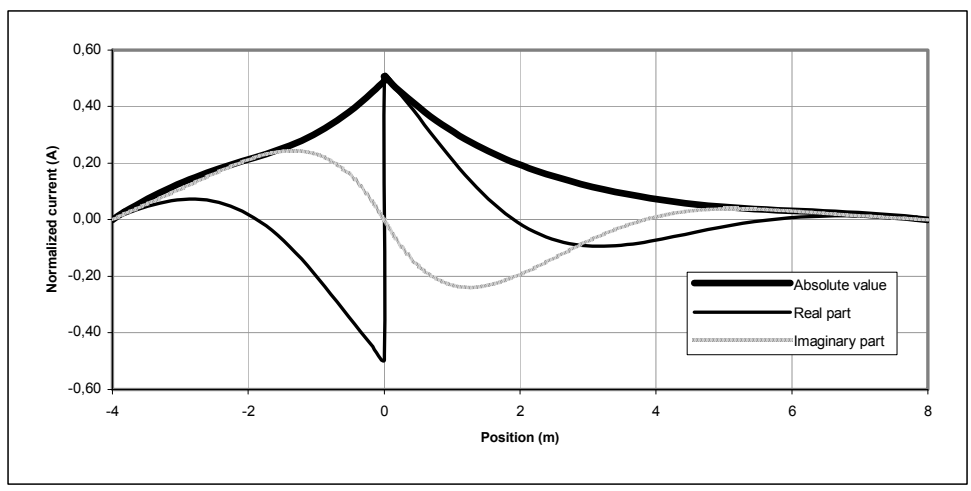

Figure 4: The current distribution along the two-wire junction: $L_{1}=4 \mathrm{~m}$, $L_{2}=8 \mathrm{~m}, d=0.5 \mathrm{~m}, \varepsilon_{\mathrm{r}}=10, \rho=100 \Omega \mathrm{m}, f=10 \mathrm{MHz}$

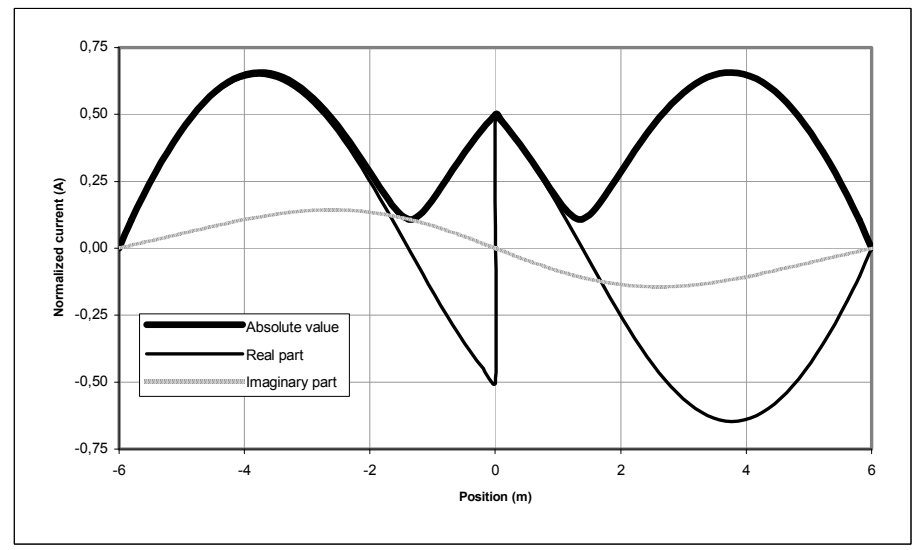

Figure 5: The current distribution along the two-wire junction: $L_{1}=6 \mathrm{~m}$, $L_{2}=6 \mathrm{~m}, d=0.5 \mathrm{~m}, \varepsilon_{\mathrm{r}}=10, \rho=5400 \Omega \mathrm{m}, f=10 \mathrm{MHz}$. 


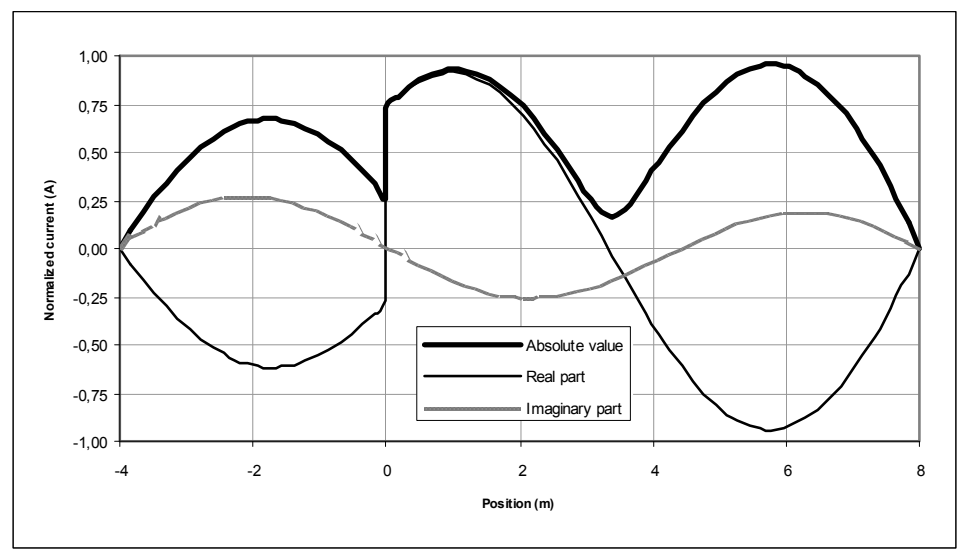

Figure 6: The current distribution along the two-wire junction: $L_{1}=4 \mathrm{~m}$, $L_{2}=8 \mathrm{~m}, d=0.5 \mathrm{~m}, \varepsilon_{\mathrm{r}}=10, \rho=5400 \Omega \mathrm{m}, f=10 \mathrm{MHz}$.

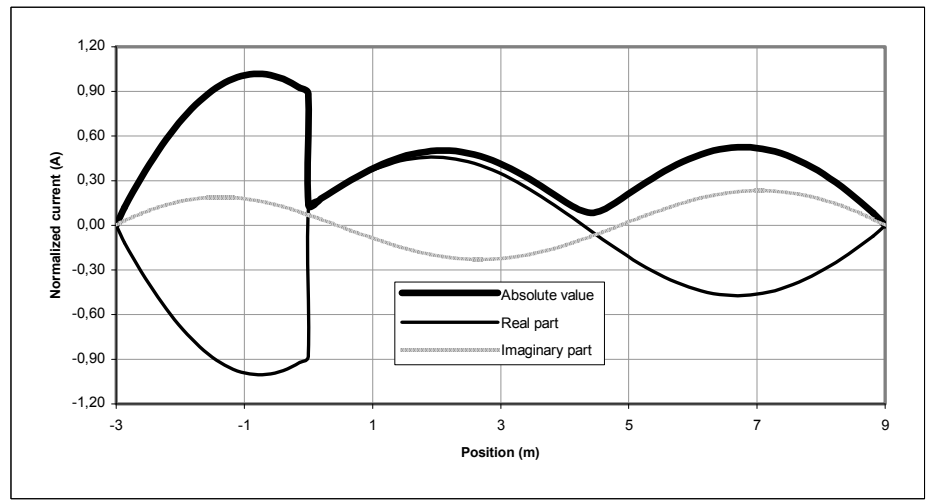

Figure 7: The current distribution along the two-wire junction: $L_{1}=3 \mathrm{~m}$, $L_{2}=9 \mathrm{~m}, d=0.5 \mathrm{~m}, \varepsilon_{\mathrm{r}}=10, \rho=5400 \Omega \mathrm{m}, f=10 \mathrm{MHz}$.

\subsection{The three-wire ( $T$ and $Y$ ) junctions}

Next set of computational examples is related to grounding systems in the form of T-junction and Y-junction, Fig. 8. The ground permittivity is $\varepsilon_{\mathrm{r}}=10$, specific resistance $\rho=1000 \Omega \mathrm{m}$, the burial depth $d=0.5 \mathrm{~m}$ and the frequency is $f=10 \mathrm{MHz}$.

The current distribution along the T-shaped grounding is shown in Fig. 9, while the Y-shaped grounding system is presented in Fig. 10. 

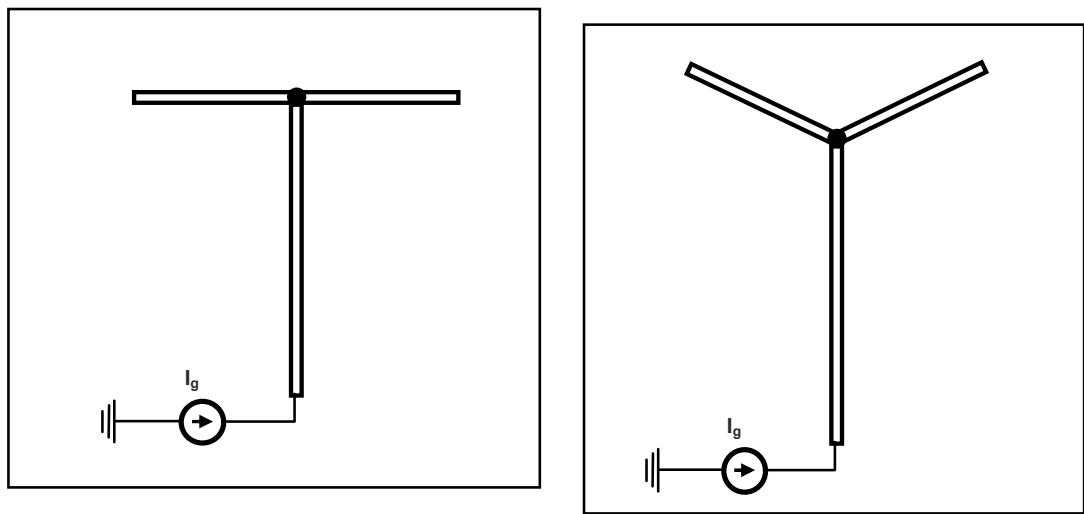

a) T-junction

b) Y-junction

Figure 8: The three wire junction energized by th ecurrent source.

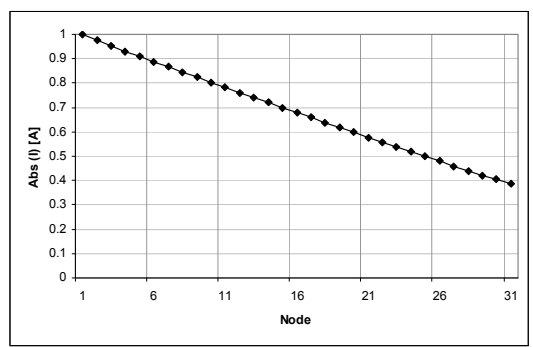

a) $L_{1}=1.5 \mathrm{~m}$

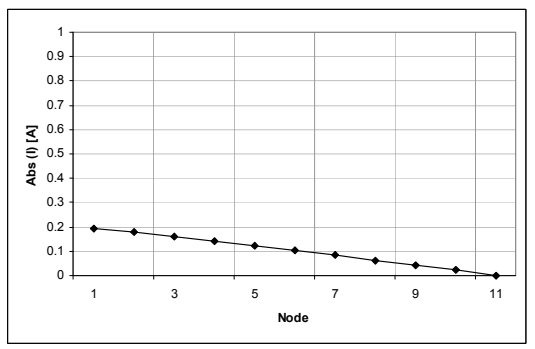

b) $L_{2}=0.5 \mathrm{~m}$

Figure 9: The current distribution along the T- junction.

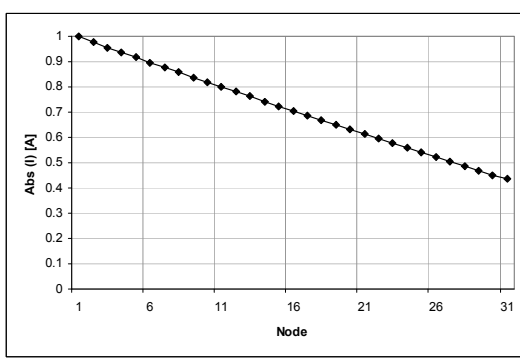

a) $L_{1}=1.5 \mathrm{~m}$

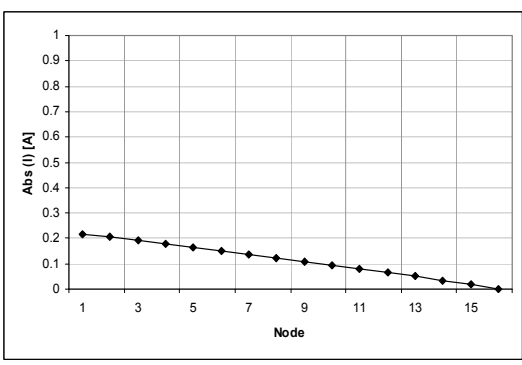

b) $L_{2}=0.56 \mathrm{~m}$

Figure 10: The current distribution along the Y-junction. 


\section{Concluding remarks}

The paper deals with an assessment of the current distribution along the complex grounding systems composed from interconnected conductors. The formulation is based on the wire antenna theory and the related set of Pocklington integrodifferential equations for wires of arbitrary shape. The set of Pocklington equations are numerically handled by using the Galerkin-Bubnov scheme of the Indirect Boundary Element Method (GB-IBEM). Some illustrative numerical results are presented in the paper.

Future work will involve the calculation of scattered voltage, input impedance and transient impedance for the case of complex grounding systems including interconnected conductors.

\section{References}

[1] Y. Liu, M. Zitnik, R. Thottappillil, “An Improved Transmission Line Model of Grounding System”, IEEE Trans. EMC, Vol.43, No.3, pp. 348-355, 2001.

[2] G. Ala, M. L. Di Silvestre, "A Simulation Model for Electromagnetic Transients in Lightning Protection Systems", IEEE Trans. EMC, Vol.44, No.4, pp.539-534, 2003.

[3] L. Grcev , F. Dawalibi, "An Electromagnetic Model for Transients in Grounding Systems”, IEEE Trans. Power Delivery, No 4., pp. 1773-1781, Oct. 1990.

[4] L. D. Grcev, F.E. Menter, "Transient Electro-magnetic Fields Near Large Earthing Systems", IEEE Trans. Magnetics, Vol. 32, pp. 1525-1528, May 1996.

[5] D. Poljak, V. Roje, "The Integral equation method for ground wire impedance", Constanda, C., Saranen, J., Seikkala, S. (Ed), Integral methods in science and engineering, Vol. I, Longman, UK., 139-143, 1997.

[6] D. Poljak, El Khamlichi Drissi, R.Goic, Boundary element modelling of horizontal grounding electrodes using the set of generalized telegrapher's equations, BEM 31, Southampton, UK, Sept. 2009.

[7] D.Poljak, V. Dorić, K. El Khamlichi Drissi, K Kerroum, I. Medić, Comparison of wire antenna and modified transmission line approach to the assessment of frequency response of horizontal grounding electrodes. // Engineering Analysis with Boundary Elements. 32 (2008), 8; 676-681.

[8] R.G. Olsen, M.C. Willis, "A Comparison of Exact and Quasi-static Methods for Evaluating Grounding Systems at High Frequencies”, IEEE Trans. Power Delivery, Vol. 11, No 2, pp. 1071-1081, April 1996.

[9] D.Poljak, "Advanced Modeling in Computational EMC “, Wiley, New York, 2007. 